\title{
Computation of forces from deformed visco-elastic biological tissues
}

\author{
José J. Muñoz ${ }^{1}$, David Amat², Vito Conte ${ }^{2}$
}

${ }^{1}$ Laboratori de Càlcul Numèric (LaCàN), Universitat Politècnica de Catalunya, Barcelona, Spain. j.munoz@upc .edu

${ }^{2}$ Mechanics of Development and Disease, Institute for Bioengineering of Catalonia, Barcelona Institute of Science and Technology, Barcelona 08028, Spain. vconte@ibecbarcelona.eu

keywords: visco-elasticity, tractions, tissue mechanics, finite elements, embryogenesis

\begin{abstract}
We present a least-squares based inverse analysis of visco-elastic biological tissues. The proposed method computes the set of contractile forces (dipoles) at the cell boundaries that induce the observed and quantified deformations. We show that the computation of these forces requires the regularisation of the problem functional for some load configurations that we study here. The functional measures the error of the dynamic problem being discretised in time with a second-order implicit time-stepping and in space with standard finite elements. We analyse the uniqueness of the inverse problem and estimate the regularisation parameter by means of a L-curved criterion. We apply the methodology to a simple toy problem and to an in vivo set of morphogenetic deformations of the Drosophila embryo.
\end{abstract}

\section{Introduction}

Mechanical forces play a crucial role in mediating genetic information from the molecular to the cellular and tissue levels [17]. Equally, mechanical cues 
at the cellular and tissue levels influence cell signalling and fate through mechanosensing and mechanotransduction processes [18, 38].

However, measuring forces in biological tissues has proved difficult, not only due to experimental challenges, but also due to the need of robust computational methods for in vitro and in vivo quantification $[9,30]$. Recent techniques such as Force Inference (FI) [7,33] and Traction Microscopy (TM) [29] have proved particularly useful to adequately describe the physical mechanisms driving cell migration and shaping biological organisms. Although both such techniques are based on the solution of an inverse problem, these problems are based on different assumptions. In the former case the material is assumed viscous with a set of distributed contractile boundaries. In the latter case, instead, where the cells deform a mechanically well characterised substrate, an inverse elastic problem is solved [2,25]. Due to the different experimental data acquired, the inverse problem is also solved differently in each case. In IF techniques, iterative recursive least-squares methods are employed $[11,26]$, while Tikhonov regularisation of the functional is commonly applied in the second case [2, 25, 28, 34].

In the present paper we aim to find the set of external loads that cause the observed deformations of a visco-elastic medium, upon assuming a particular load discretisation. We do not intend here to determine the material parameters [5], detect cracks [1,3], or identify boundary portions [22]. Instead, the present article expands on previous works in inverse static analysis [25] and on the Cauchy problem [21,39]. The novelty of the present work consists on three main contributions. First, we combine elastic and viscous material rheology to solve the visco-elastic inverse problem, since embryonic tissues have shown to exhibit elastic behaviour on short time-scales and viscous behaviour on longer time-scales [12, 23]. Second, we extend the inverse analysis to time-dependent problems. We include a time-discretisation of the governing equations which we solve at different time-points. And third, we solve the inverse problem for two different load discretisations: traction contributions at nodes and contractile dipoles specified along edge lines.

As shown in this work, the type of load considered (body load, point load or surface load) and its location, which we call load topology, impacts the uniqueness of the solution and the degree of regularisation of the inverse problem. Furthermore, the capability of the method to search for these different load topologies is of key importance to maximise experimental applicability. Nodal forces have been used in purely elastic inverse problems via Traction Microscopy $[2,34,35]$ in order to simulate wound healing [8] 
and collective invasion [31] in living tissues. Likewise, cortical contractility along epithelial cell boundaries has been shown to drive deformations of embryonic tissues during morphogenesis [16]. The solution of a purely viscous inverse problem based on contractile forces can be found in [11], where the authors resort to a modified least-squares method that adopts a forgetting factor [26]. Similar purely viscous inverse problems have also been analysed in the frequency domain [14].

In Section 2 we introduce the formulation, notation and discretisation of the direct problem. In Section 3 we present the inverse problem and the Tikhonov regularisation of a functional that measures the error in the discrete visco-elastic equilibrium in conjunction with a L-curve criterion to determine the regularisation parameter. We apply the methodology in Section 4, either to a toy problem and to a cluster of cells during the morphogenesis of the early Drosophila embryo.

\section{Direct visco-elasticity problem}

\subsection{Continuous problem}

We consider an elastic body occupying an open connected domain $\Omega \subset \mathbb{R}^{d}$ ( $d=$ number of space dimensions) and bounded by a Lipschitz-continuous surface $\Gamma=\partial \Omega$. Let us describe the deformation with a vector field $\boldsymbol{u}(\boldsymbol{x}, t)$, with $\boldsymbol{x} \in \Omega$ and $t>0$ a time parameter. We shall here focus on small strain problems, in which case $\|\nabla \boldsymbol{u}(\boldsymbol{x}, t)\| \ll 1$. The body is subjected to boundary loads $\boldsymbol{g}(\boldsymbol{x}, t)$ on a region $\Gamma_{g} \subset \Gamma$ and to specific displacements $\hat{\boldsymbol{u}}(\boldsymbol{x}, t)$ on $\hat{\Gamma}=\Gamma \backslash \Gamma_{g}$. By neglecting inertial forces, the displacement field is given by the solution of the initial value problem [10],

$$
\begin{aligned}
\nabla \cdot \boldsymbol{\sigma}(\boldsymbol{x}, t) & =\mathbf{0}, & & \boldsymbol{x} \in \operatorname{int}(\Omega), t \in[0, T], \\
\boldsymbol{\sigma}(\boldsymbol{x}, t) \boldsymbol{n} & =\boldsymbol{g}(\boldsymbol{x}, t), & & \boldsymbol{x} \in \Gamma_{g}, t \in[0, T], \text { (Newmann condition) } \\
\boldsymbol{u}(\boldsymbol{x}, t) & =\hat{\boldsymbol{u}}(\boldsymbol{x}, t), & & \boldsymbol{x} \in \hat{\Gamma}, t \in[0, T], \text { (Dirichlet condition) } \\
\boldsymbol{u}(\boldsymbol{x}, 0) & =\boldsymbol{u}_{0}(\boldsymbol{x}), & & \boldsymbol{x} \in \Omega, \text { (initial condition) } .
\end{aligned}
$$

The last equation is the initial conditions, with $\boldsymbol{u}_{0}(\boldsymbol{x})$ a given initial displacement. The stress tensor $\boldsymbol{\sigma}(\boldsymbol{x}, t)$ is related to the displacement field through a visco-elastic constitutive relation, which we assume of the Maxwell type,

$$
\boldsymbol{\sigma}(\boldsymbol{x}, t)=\boldsymbol{\sigma}^{e}(\boldsymbol{x}, t)+\boldsymbol{\sigma}^{v}(\boldsymbol{x}, t),
$$


with

$$
\begin{aligned}
& \boldsymbol{\sigma}^{e}(\boldsymbol{x}, t)=\lambda_{L} \operatorname{tr}(\boldsymbol{\varepsilon}[\boldsymbol{u}]) \mathbf{I}+2 \mu_{L} \boldsymbol{\varepsilon}[\boldsymbol{u}] \\
& \boldsymbol{\sigma}^{v}(\boldsymbol{x}, t)=\eta \boldsymbol{\varepsilon}^{\operatorname{dev}}[\dot{\boldsymbol{u}}]
\end{aligned}
$$

and

$$
\begin{aligned}
\boldsymbol{\varepsilon}[\boldsymbol{u}] & =\frac{1}{2}\left(\nabla \boldsymbol{u}+\nabla \boldsymbol{u}^{T}\right), \\
\varepsilon^{\operatorname{dev}}[\dot{\boldsymbol{u}}] & =\boldsymbol{\varepsilon}[\dot{\boldsymbol{u}}]-\frac{1}{d}(\nabla \cdot \dot{\boldsymbol{u}}) \mathbf{I} .
\end{aligned}
$$

For the sake of clarity, we have removed the dependence of $\boldsymbol{u}, \dot{\boldsymbol{u}}, \boldsymbol{\varepsilon}$ and $\varepsilon^{d e v}$ on $\boldsymbol{x}$ and $t$. A superimposed dot denotes time differentiation, and the Lamé material parameters $\left(\lambda_{L}\right.$ and $\left.\mu_{L}\right)$, and the viscous coefficient $(\eta)$ are considered as known a priori and constant. Note that the viscous stresses are proportional to the deviatoric strains, which is due to the fact that the spherical part of the viscous response can be neglected in fluids and viscoelastic materials [20, Section 6.4].

Remark 1. In cellular active materials, and in addition to $\boldsymbol{\sigma}^{e}$ and $\boldsymbol{\sigma}^{v}$, we may also consider a contractile stress $\boldsymbol{\sigma}^{c}$, consisting on the active forces at the cell boundary (cortex and junctional forces). We express these stresses as $\boldsymbol{\sigma}^{c}=c(\boldsymbol{x}) \delta\left(\boldsymbol{x}-\boldsymbol{x}^{c}\right) \boldsymbol{\tau} \otimes \boldsymbol{\tau}$, where $\delta(\boldsymbol{x})$ is the Dirac delta function, $c(\boldsymbol{x})$ a contractility intensity, $\boldsymbol{x}^{c}$ the location of the cell boundary, and $\boldsymbol{\tau}$ is the direction of the force, tangent to the cell membrane.

\subsection{Weak form}

In order to present the time- and spacial-discretisation of the initial value problem in (1)-(3), let us define the following spaces $U$ and $V$,

$$
\begin{gathered}
=H^{1}(\Omega)^{d} \\
V=\left(H_{0}^{1}(\Omega)\right)^{d}=\left\{\boldsymbol{v} \in\left(H^{1}(\Omega)\right)^{d}: v_{i}=0 \text { on } \hat{\Gamma}, i=1,2,3\right\},
\end{gathered}
$$

endowed with the scalar product $(\boldsymbol{u}, \boldsymbol{v})=\int_{\Omega} \boldsymbol{u} \cdot \boldsymbol{v} d \Omega$, and equipped with norm $\|\boldsymbol{v}\|_{1, \Omega}^{2}=\int_{\Omega}\left(\left|v_{i}\right|^{2}+\nabla v_{i} \cdot \nabla v_{i}\right) d \Omega$. After multiplying by a trial function $\boldsymbol{v} \in V$ the first equation in (2), integrating by parts and exploiting the symmetry of the stress tensor, the solution $\boldsymbol{u}$ of may be found as the vector field that satisfies the weak form

$$
\text { Find } \boldsymbol{u} \in U \text { such that, } a^{e}(\boldsymbol{u}, \boldsymbol{v})+a^{v}(\boldsymbol{u}, \boldsymbol{v})=l(\boldsymbol{v}), \forall \boldsymbol{v} \in V \text {, }
$$


where the bilinear and linear forms $a^{e}(),, a^{v}($,$) and b()$ are given by,

$$
\begin{aligned}
a^{e}(\boldsymbol{u}, \boldsymbol{v}) & =\int_{\Omega} \boldsymbol{\varepsilon}[\boldsymbol{v}]: \boldsymbol{\sigma}^{e} d \Omega, \\
a^{v}(\boldsymbol{u}, \boldsymbol{v}) & =\int_{\Omega} \boldsymbol{\varepsilon}[\boldsymbol{v}]: \boldsymbol{\sigma}^{v} d \Omega, \\
l(\boldsymbol{v}) & =\int_{\Gamma_{g}} \boldsymbol{v} \cdot \boldsymbol{g}(\boldsymbol{x}, t) d \Gamma .
\end{aligned}
$$

The traction field $\boldsymbol{g}(\boldsymbol{x}, t)$ may be discontinuous, but we assume that $\boldsymbol{g}(\boldsymbol{x}, t) \in G \subseteq L^{2}\left(\Gamma_{g}\right)$.

Remark 2. Following Remark 2, by including the contractile stresses $\boldsymbol{\sigma}^{c}$ we would obtain an additional term in the weak form:

$$
l^{c}(\boldsymbol{v})=\int_{\Omega} \boldsymbol{\sigma}^{c}: \boldsymbol{\varepsilon}[\boldsymbol{v}] d \Omega=\int_{\Gamma^{c}} c(\boldsymbol{x}) \boldsymbol{\tau} \cdot \nabla \boldsymbol{v} \boldsymbol{\tau} d \Gamma .
$$

\subsection{Time-discretisation}

In order to ease the solution of time-dependent inverse problems, we will resort to a partition of the interval of interest $[0, T]$ into a set of $N+1$ timediscrete instants $t_{0}, t_{1}, \ldots, t_{N}$, and to a simple $\theta$-weighted time-stepping of the displacement as,

$$
\begin{array}{r}
\dot{\boldsymbol{u}} \approx \frac{\boldsymbol{u}_{n+1}-\boldsymbol{u}_{n}}{h}, \\
\boldsymbol{u} \approx(1-\theta) \boldsymbol{u}_{n}+\theta \boldsymbol{u}_{n+1}=: \boldsymbol{u}_{n+\theta},
\end{array}
$$

where $\boldsymbol{u}_{n}(\boldsymbol{x}) \approx \boldsymbol{u}\left(\boldsymbol{x}, t_{n}\right)$, and $h=t_{n+1}-t_{n}$ is a constant time-step size. By using this approximation, the weak form in (5) turns into

Find $\boldsymbol{u} \in U$ such that, $a^{e}\left(\boldsymbol{u}_{n+\theta}, \boldsymbol{v}\right)+a^{v}\left(\boldsymbol{u}_{n+\theta}, \boldsymbol{v}\right)=l_{n+\theta}(\boldsymbol{v}), \forall \boldsymbol{v} \in V$, with

$$
\begin{aligned}
a^{e}\left(\boldsymbol{u}_{n+\theta}, \boldsymbol{v}\right) & =\int_{\Omega} \boldsymbol{\varepsilon}[\boldsymbol{v}]: \boldsymbol{\sigma}_{n+\theta}^{e} d \Omega \\
a^{v}\left(\boldsymbol{u}_{n+\theta}, \boldsymbol{v}\right) & =\int_{\Omega} \boldsymbol{\varepsilon}[\boldsymbol{v}]: \boldsymbol{\sigma}_{n+\theta}^{v} d \Omega \\
l_{n+\theta}(\boldsymbol{v}) & =\int_{\Gamma_{g}} \boldsymbol{v} \cdot \boldsymbol{g}_{n+\theta} d \Gamma
\end{aligned}
$$

and

$$
\begin{array}{r}
\boldsymbol{\sigma}_{n+\theta}^{e}=\lambda_{L}\left(\nabla \cdot \boldsymbol{u}_{n+\theta}\right) \mathbf{I}+\mu_{L}\left(\nabla \boldsymbol{u}_{n+\theta}+\nabla \boldsymbol{u}_{n+\theta}^{T}\right) \\
\boldsymbol{\sigma}_{n+\theta}^{v}=\frac{\eta}{h}\left(\varepsilon^{\operatorname{dev}}\left[\boldsymbol{u}_{n+1}\right]-\boldsymbol{\varepsilon}^{\operatorname{dev}}\left[\boldsymbol{u}_{n}\right]\right) \\
\boldsymbol{g}_{n+\theta}=(1-\theta) \boldsymbol{g}\left(\boldsymbol{x}, t_{n}\right)+\theta \boldsymbol{g}\left(\boldsymbol{x}, t_{n+1}\right) .
\end{array}
$$




\subsection{Finite element spatial discretisation}

We will consider a spatial discretisation of the weak form in (8)-(10) by using a mesh $M$ consisting on a partition of the domain $\Omega$ into $N_{E}$ non-overlapping conformal elements $K_{1}, \ldots, K_{N_{E}}$ and $N_{n}$ nodes $\boldsymbol{x}_{i} \in \Omega, i=1,2, \ldots, N_{n}$. Elements $K_{e}, e=1, \ldots, N_{E}$ are such that [4]

$$
\bar{\Omega}=K_{1} \cup K_{2} \ldots \cup K_{E}, \operatorname{int}\left(K_{e}\right) \cap \operatorname{int}\left(K_{e^{\prime}}\right)=\emptyset, \forall e \neq e^{\prime} .
$$

We will resort to the polynomial basis $Q_{r}\left(K_{e}\right)$ on an element $K_{e}$,

$$
Q_{r}\left(K_{e}\right)=\left\{q: q(x, y)=\sum_{0 \leq i, j \leq r} c_{i j} x^{i} y^{j},(x, y) \in K_{e}, c_{i j} \in \mathbb{R}, \forall K_{e} \in M\right\} .
$$

The basis is defined in a two-dimensional space $(d=2)$, but this can be extended to three dimensions without altering the conclusions and methodology described henceforth. Also, in the numerical example reported in Section 4 , we will focus on the case $r=1$, which is equivalent to using the finite element spaces $U^{h} \subset U$ and $V^{h} \subset V$ defined as

$$
\begin{aligned}
U^{h} & =\left\{\boldsymbol{v}(\boldsymbol{x}) \in H^{1}(\Omega)^{d}: \boldsymbol{v} \in C(\Omega),\left.\boldsymbol{v}\right|_{K_{e}} \in Q_{1}\left(K_{e}\right), \forall K_{e} \in M\right\}, \\
V^{h} & =\left\{\boldsymbol{v}(\boldsymbol{x}) \in H_{0}^{1}(\Omega)^{d}: \boldsymbol{v} \in C(\Omega),\left.\boldsymbol{v}\right|_{K_{e}} \in Q_{1}\left(K_{e}\right), \forall K_{e} \in M\right\} .
\end{aligned}
$$

As yet, we will assume that the boundary loads $\boldsymbol{g}$ belong to a set $G$ of piece-wise linear functions in $C^{0}\left(\Gamma_{g}\right)$,

$$
G^{h}=\left\{\boldsymbol{g}(\boldsymbol{x}) \in\left(L^{2}\left(\Gamma_{g}\right)\right)^{d}:\left.\boldsymbol{g}\right|_{\partial K_{e}} \in Q_{1}\left(\partial K_{e}\right), \forall \partial K_{e} \in \Gamma_{e}\right\}
$$

The displacement and traction spaces $U^{h}$ and $G^{h}$ allow us to express the interpolated fields at each time-step $t_{n}$ in terms of their bases $q_{j}(\boldsymbol{x})$ and $\bar{q}_{j}(\boldsymbol{x})$, respectively as,

$$
\begin{array}{r}
\boldsymbol{u}_{n} \approx \boldsymbol{u}_{n}^{h}=\sum_{\forall j} q_{j}(\boldsymbol{x}) \mathbf{u}_{n}^{j} \\
\boldsymbol{g}_{n} \approx \boldsymbol{g}_{n}^{h}=\sum_{\forall j, \boldsymbol{x}_{j} \in \Gamma_{g}} \bar{q}_{j}(\boldsymbol{x}) \mathbf{g}_{n}^{j},
\end{array}
$$

with $\mathbf{u}_{j} \in \mathbb{R}^{d}$ and $\mathbf{g}_{j} \in \mathbb{R}^{d}$ the nodal displacements and tractions at time $t_{n}$. By replacing the continuous field $\boldsymbol{u}_{n}$ and $\boldsymbol{g}_{n}$ by their discrete counterpart $\boldsymbol{u}_{n}^{h}$ 
and $\boldsymbol{g}_{n}^{h}$, and imposing the weak form on all $\boldsymbol{v}^{h} \in V^{h}$ instead of $\boldsymbol{v} \in V$, the discrete solution $\mathbf{u}_{n+1}^{T}=\left\{\mathbf{u}_{n+1}^{0}{ }^{T} \ldots \mathbf{u}_{n+1}^{N_{n} T}\right\}$ is found by solving the following system of equations,

$$
\mathbf{K} \mathbf{u}_{n+\theta}+\mathbf{D} \frac{\mathbf{u}_{n+1}-\mathbf{u}_{n}}{h}=\tilde{\mathbf{G}} \tilde{\mathbf{g}}_{n+\theta}
$$

where the stiffness matrix $\mathbf{K}$, damping matrix $\mathbf{D}$ and loading matrix $\tilde{\mathbf{G}}$ are given by,

$$
\begin{array}{r}
\mathbf{K}_{i j}=\int_{\Omega}\left(\lambda_{L} \nabla q_{i} \otimes \nabla q_{j}+\mu_{L}\left(\left(\nabla q_{i} \cdot \nabla q_{j}\right) \mathbf{I}+\nabla q_{j} \otimes \nabla q_{i}\right)\right) d \Omega \\
\mathbf{D}_{i j}=\int_{\Omega}\left(\frac{\eta}{2}\left(\nabla q_{j} \otimes \nabla q_{i}+\left(\nabla q_{i} \cdot \nabla q_{j}\right) \mathbf{I}\right)-\frac{\eta}{d} \nabla q_{i} \otimes \nabla q_{j}\right) d \Omega, \\
\tilde{\mathbf{G}}_{i j}=\int_{\Gamma_{g}} q_{i} \bar{q}_{j} \mathbf{I} d \Gamma .
\end{array}
$$

In further manipulations, it will be convenient to factorise matrix $\tilde{\mathbf{G}}$ as $\tilde{\mathbf{G}}=\mathbf{G M}$, with $\mathbf{M}$ the standard mass matrix of boundary $\Gamma_{g}$ with a unit density and $\mathbf{G}$ a matrix with solely 0 s and $1 \mathrm{~s}$,

$$
\begin{aligned}
\mathbf{M}_{i j} & =\int_{\Gamma_{g}} \bar{q}_{i} \bar{q}_{j} \mathbf{I} d \Gamma, \\
\mathbf{G}_{i j} & =\left\{\begin{array}{l}
1 \text { if global node } i \text { corresponds to node } j \text { at } \Gamma_{g}, \\
0 \text { otherwise }
\end{array}\right.
\end{aligned}
$$

which allows us to rewrite equation (11) as,

$$
\mathbf{K} \mathbf{u}_{n+\theta}+\mathbf{D} \frac{\mathbf{u}_{n+1}-\mathbf{u}_{n}}{h}=\mathbf{G g}_{n+\theta}
$$

with

$$
\mathbf{g}_{n+\theta}=\mathbf{M} \tilde{\mathbf{g}}_{n+\theta} .
$$

The nodal contributions $\tilde{\mathbf{g}}_{n+\theta}$ may be computed from $\mathbf{g}_{n+\theta}$ as $\tilde{\mathbf{g}}_{n+\theta}=$ $\mathbf{M}^{-1} \mathbf{g}_{n+\theta}$, which is always well-defined since $\mathbf{M}$ is positive definite. Physically, the components $\mathbf{g}_{n+\theta}$ correspond to nodal forces, while $\tilde{\mathbf{g}}_{n+\theta}$ correspond to nodal tractions (forces per unit of surface).

We also note that the case $\theta=\frac{1}{2}$, which is the numerical value that we will use in a our numerical results in Section 4, corresponds to a standard Crank-Nicholson time-discretisation, yielding a second order implicit method [19]. 
Remark 3. Matrices $\mathbf{D}$ and $\mathbf{K}$ are semi-positive definite, and $\operatorname{dim}(\operatorname{ker}(\mathbf{D}))>$ $\operatorname{dim}(\operatorname{ker}(\mathbf{K}))$.

This can be verified by setting $\|\varepsilon(\boldsymbol{u})\|^{2}=\varepsilon(\boldsymbol{u}): \varepsilon(\boldsymbol{u})$ and noting that for a given interpolated displacement $\mathbf{u}$, we have that

$$
\begin{aligned}
\mathbf{u} \cdot \mathbf{K u} & =\int_{\Omega} \boldsymbol{\varepsilon}\left[\boldsymbol{u}^{h}\right]: \boldsymbol{\sigma}\left(\boldsymbol{u}^{h}\right) d \Omega \\
& =\int_{\Omega}\left(\lambda_{L}\left(\operatorname{tr}\left(\boldsymbol{\varepsilon}\left(\boldsymbol{u}^{h}\right)\right)\right)^{2}+2 \mu_{L}\left\|\boldsymbol{\varepsilon}\left(\boldsymbol{u}^{h}\right)\right\|^{2}\right) d \Omega \geq 0, \\
\mathbf{u} \cdot \mathbf{D u} & =\int_{\Omega} \eta \varepsilon^{\operatorname{dev}}\left[\boldsymbol{u}^{h}\right]: \boldsymbol{\varepsilon}^{\operatorname{dev}}\left[\boldsymbol{u}^{h}\right] d \Omega \\
& =\eta \int_{\Omega}\left\|\varepsilon^{\operatorname{dev}}\left[\boldsymbol{u}^{h}\right]\right\|^{2} d \Omega \geq 0 .
\end{aligned}
$$

The equality in (16) holds whenever $\boldsymbol{\varepsilon}\left(\boldsymbol{u}^{h}\right)=\mathbf{0}$, which corresponds to a rigid body motion. If the boundary conditions of the problems are wellposed, and no trivial solution is allowed, these motions are prevented. In equation (17), the identity holds whenever $\varepsilon^{d e v}\left[\boldsymbol{u}^{h}\right]=0$, that is, whenever a rigid motion is allowed and thus $\varepsilon\left[\boldsymbol{u}^{h}\right]=\mathbf{0}$, but also whenever $\varepsilon\left[\boldsymbol{u}^{h}\right]=$ $\frac{1}{d} \operatorname{tr}\left(\varepsilon\left[\boldsymbol{u}^{h}\right]\right) \mathbf{I}$, which corresponds to an isotropic deformation. Due to this additional condition, we deduce that $\operatorname{dim}(\operatorname{ker}(\mathbf{D}))>\operatorname{dim}(\operatorname{ker}(\mathbf{K}))$.

\subsection{Solving for contractile forces dipoles}

Cell boundaries are able to exert contractile forces which form a set of selfequilibrated force dipoles. For this reason, we set out to rewrite the system of equations in correspondence with this new load configuration. We express the loading term in the right hand side of (11) as a set of $n^{c}$ pairs of forces with equal magnitude and opposite direction. As such, the load can be parametrised through a vector $\mathbf{c}^{T}=\left\{c_{1}, \ldots, c_{n^{c}}\right\}$ where each component $c_{\alpha}$ is associated to an element boundary between two nodes, let's say $\boldsymbol{x}_{i}$ and $\boldsymbol{x}_{j}$, and the value $c_{\alpha}$ is the intensity of the contractile force. Consequently, each scalar $c_{\alpha}$ represents two opposing contractile forces of each dipole $c_{\alpha} \boldsymbol{e}_{\alpha}^{i}=$ $-c_{\alpha} \boldsymbol{e}_{\alpha}^{j}$, with $\boldsymbol{e}_{\alpha}^{i}=\left(\boldsymbol{x}_{j}-\boldsymbol{x}_{i}\right) /\left\|\boldsymbol{x}_{j}-\boldsymbol{x}_{i}\right\|$, as illustrated in Figure 1 .

The resulting set of nodal forces can be expressed as the product $\mathbf{C c}$, where the load matrix $\mathbf{C} \in \mathbb{R}^{\left(N_{n} * d\right) \times n^{c}}$ is given by,

$$
\mathbf{C}_{i \alpha}= \begin{cases}0 & \text { if contraction } c_{\alpha} \text { is not applied on node } i \\ \boldsymbol{e}_{\alpha}^{i} & \text { if contraction } c_{\alpha} \text { is applied on node } i\end{cases}
$$




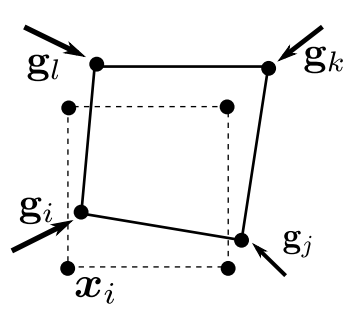

(a)

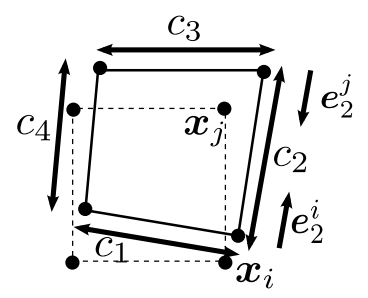

(b)

Figure 1: Illustration of type of loading in one element. (a) Nodal contributions $\mathbf{g}_{i}, \ldots, \mathbf{g}_{l}$ due to external surface tractions. (b) Contractile loads (dipoles) $c_{1}, \ldots, c_{4}$.

With this new type of contractile dipoles, the discrete direct problem in (11) turns into,

$$
\mathbf{K} \mathbf{u}_{n+\theta}+\mathbf{D} \frac{\mathbf{u}_{n+1}-\mathbf{u}_{n}}{h}=\mathbf{C} \mathbf{c}_{n+\theta}
$$

We recognise that due to the configuration of the contractile forces, which are applied along element boundaries, our load configuration is strongly mesh dependent. However, we note that biological materials are discrete in nature. In fact, in our examples, the mesh will be adapted to the cell boundaries, and we will assume contractile forces only on a subset of all the element boundaries.

Remark 4. A similar set of dipoles would be obtained following the discretisation of the contractile stresses $\boldsymbol{\sigma}^{c}$ introduced in Remarks 1 and 2. Indeed, after using the discretisation of $\boldsymbol{v}$ through the space $V^{h}$, and considering a constant contractility factor $c_{i j}$ along each boundary segment between nodes $\boldsymbol{x}_{i}$ and $\boldsymbol{x}_{j}$, the force term $l^{c}(\boldsymbol{v})$ in (6) becomes,

$$
l_{i j}^{c}(\boldsymbol{v})=\left(\boldsymbol{v}^{j}-\boldsymbol{v}^{i}\right) \cdot \boldsymbol{\tau}^{i j} c_{i j}
$$

Here, we have used the fact that $\nabla \boldsymbol{v}=\sum_{i} \nabla q_{i} \otimes \boldsymbol{v}^{i}$ and therefore, along each segment ij we have $\boldsymbol{\tau} \cdot(\nabla \boldsymbol{v}) \boldsymbol{\tau}=\boldsymbol{\tau}^{i j} \cdot\left(\boldsymbol{v}^{j}-\boldsymbol{v}^{i}\right) / L^{i j}$, with $L^{i j}=\left\|\boldsymbol{x}^{i}-\boldsymbol{x}^{j}\right\|$. By gathering all the contributions $l_{i j}^{c}(\boldsymbol{v})$, the weak form using $\boldsymbol{\sigma}^{c}$ would then yield equivalent equations to the ones obtained using the contractile force dipoles. 


\section{Inverse problem}

\subsection{Measured deformations}

The main objective of the inverse method presented here is to develop a technique that allows computing the most plausible forces from a set of observed deformations on a linear visco-elastic material. We will thus assume that mechanical equilibrium of this material is governed by the discrete equilibrium equations in (11) or (19).

In practice though, the displacements can only be retrieved for some of the nodes of mesh $M$. While some authors have derived methods for arbitrary locations of the measured displacements [34], we will assume here that a set of nodal displacements $\mathbf{u}_{n+1}^{A}$ are measured on a domain $\Omega^{A}$, and that the remaining displacements $\mathbf{u}_{n+1}^{B}$ are unknown. The inverse method presented here will compute both the forces $\mathbf{g}_{n+1}$ (or contractions $\mathbf{c}_{n+1}$ ) producing the observed deformations $\mathbf{u}_{n+1}^{A}$, and the unknown displacements $\mathbf{u}_{n+1}^{B}$ on $\Omega \backslash \Omega^{A}$. We will also assume that $\mathbf{u}_{n}^{A}, \mathbf{u}_{n}^{B}$ and $\mathbf{g}_{n}$ (or $\mathbf{c}_{n}$ ) are all known, that the domains $\Omega^{A}, \Omega^{B}$ and $\Gamma_{g}$ do not change in time, and that $\operatorname{int}\left(\Omega^{A}\right) \cap \operatorname{int}\left(\Omega^{B}\right)=$ $\emptyset$ with no node belonging to the two sets. We note that we are in fact considering the partition of the space $U^{h}$ in the direct sum

$$
U^{h}=U^{A} \oplus U^{B},
$$

with $\mathbf{u}^{A} \in U^{A}$ and $\mathbf{u}^{B} \in U^{B}$. Finally, we also consider that the measured displacements $\mathbf{u}^{A}$ prevent the presence of undetermined rigid body motions on the whole domain $\Omega$.

It will become convenient in subsequent derivations to split the columns of matrices $\mathbf{K}$ and $\mathbf{D}$ in (12) into those associated to $\mathbf{u}^{A}$ and $\mathbf{u}^{B}$,

$$
\begin{aligned}
& \mathbf{K} \mathbf{u}=\mathbf{K}^{A} \mathbf{u}^{A}+\mathbf{K}^{B} \mathbf{u}^{B}, \\
& \mathbf{D} \mathbf{u}=\mathbf{D}^{A} \mathbf{u}^{A}+\mathbf{D}^{B} \mathbf{u}^{B},
\end{aligned}
$$

where $\left[\begin{array}{ll}\mathbf{K}^{A} & \mathbf{K}^{B}\end{array}\right]=\mathbf{K}$ and $\left[\begin{array}{ll}\mathbf{D}^{A} & \mathbf{D}^{B}\end{array}\right]=\mathbf{D}$ are, respectively, the block-matrix representation of $\mathbf{K}$ and $\mathbf{D}$ with respect to $\mathbf{u}^{A}$ and $\mathbf{u}^{B}$, which are such that $\mathbf{u}^{T}=\left[\mathbf{u}^{A^{T}} \mathbf{u}^{B^{T}}\right]$. Let us also introduce the following notation, which will become useful in subsequent derivations,

$$
\begin{gathered}
n^{A}=\operatorname{dim}\left(U^{A}\right), n^{B}=\operatorname{dim}\left(U^{B}\right), n=n^{A}+n^{B} \\
n^{g} \quad=\operatorname{dim}\left(G^{h}\right)=\text { number of columns of } \mathbf{G} \\
n^{c} \quad=\operatorname{dim}(C)=\text { number of columns of } \mathbf{C}
\end{gathered}
$$


which allows us to specify the dimensions of the rectangular matrices in (23) as $\mathbf{A} \in \mathbb{R}^{n \times n^{B}}, \mathbf{G} \in \mathbb{R}^{n \times n^{g}}, \mathbf{C} \in \mathbb{R}^{n \times n^{c}}$.

\subsection{Nodal forces}

We assume here a set of external nodal forces, and thus that mechanical equilibrium is governed by equation (11). The inverse problem is solved by minimising, for a given $\mathbf{u}^{A}$, the following regularised quadratic functional,

$$
J_{g}\left(\mathbf{u}_{n+1}^{B}, \mathbf{g}_{n+1}\right)=\left\|\mathbf{K} \mathbf{u}_{n+\theta}+\frac{1}{h} \mathbf{D}\left(\mathbf{u}_{n+1}-\mathbf{u}_{n}\right)-\mathbf{G g}_{n+\theta}\right\|^{2}+\lambda\left\|\mathbf{g}_{n+1}\right\|^{2} .
$$

Upon resorting to the matrix partitioning in (21), the minimisation of $J_{g}\left(\mathbf{u}_{n+1}^{B}, \mathbf{g}_{n+1}\right)$ yields the following system of equations,

$$
\begin{array}{r}
\left(\left[\begin{array}{ll}
\mathbf{A} & -\theta \mathbf{G}
\end{array}\right]^{T}\left[\begin{array}{ll}
\mathbf{A} & -\theta \mathbf{G}
\end{array}\right]+\lambda \mathbf{I}_{2}^{T} \mathbf{I}_{2}\right)\left\{\begin{array}{l}
\mathbf{u}_{n+1}^{B} \\
\mathbf{g}_{n+1}
\end{array}\right\} \\
=\left[\begin{array}{ll}
\mathbf{A} & -\theta \mathbf{G}
\end{array}\right]^{T}\left(\mathbf{b}+(1-\theta) \mathbf{G g}_{n}\right)
\end{array}
$$

with

$$
\begin{array}{cc}
\mathbf{A}= & \left(\frac{1}{h} \mathbf{D}^{B}+\theta \mathbf{K}^{B}\right), \\
\mathbf{b}= & \left(\frac{1}{h} \mathbf{D}^{B}-(1-\theta) \mathbf{K}^{B}\right) \mathbf{u}_{n}^{B}-\frac{1}{h} \mathbf{D}^{A}\left(\mathbf{u}_{n+1}^{A}-\mathbf{u}_{n}^{A}\right)-\mathbf{K}^{A} \mathbf{u}_{n+\theta}^{A}, \\
\mathbf{I}_{2} & =\left[\begin{array}{ll}
\mathbf{0} & \mathbf{I}
\end{array}\right] .
\end{array}
$$

In the equations above, we have assumed that $\theta>0$. For the particular case $\theta=0$, similar equations for retrieving $\mathbf{u}_{n+1}^{B}$ and $\mathbf{g}_{n}$ from $\mathbf{u}_{n}^{B}$ and $\mathbf{u}_{n}^{A}$ can be rewritten.

It is important noticing that functional $J_{g}\left(\mathbf{u}_{n+1}^{B}, \mathbf{g}_{n+1}\right)$ uses a Tikhonov regularisation. As previously observed [25], such a regularisation may be redundant when searching for a unique solution in the purely elastic case $(\eta=0)$ or in correspondence of specific domains $\Gamma_{g}$ and $\Omega^{A}$. We will here extend these results for the viscous case $(\eta>0)$.

Remark 5. The solution of the normal equations in (23) is equivalent to

$$
\begin{array}{r}
\mathbf{A}^{T} \mathbf{J}_{g} \mathbf{A} \mathbf{u}_{n+1}^{B}=\mathbf{A}^{T} \mathbf{J}_{g}\left(\mathbf{b}-(1-\theta) \mathbf{G} \mathbf{g}_{n}\right) \\
\mathbf{g}_{n+1}=\frac{\theta}{\theta^{2}+\lambda} \mathbf{G}^{T}\left(\mathbf{A} \mathbf{u}_{n+1}^{B}-\mathbf{b}-(1-\theta) \mathbf{G} \mathbf{g}_{n}\right)
\end{array}
$$

where

$$
\mathbf{J}_{g}=\mathbf{I}-\theta^{2} \mathbf{G}\left(\theta^{2} \mathbf{G}^{T} \mathbf{G}+\lambda \mathbf{I}\right)^{-1} \mathbf{G}^{T}=\mathbf{I}-\frac{\theta^{2}}{\theta^{2}+\lambda} \mathbf{G G}^{T}
$$


System in (25) can be deduced upon replacing $\mathbf{g}_{n+1}$ in the first block of equations in (23) the expression deduced in the second block of (23). Advantageously, system in (25) is a smaller system, is better condicioned (no mixing of load and displacements fields) and allows the computation of $\mathbf{g}_{n+1}$ through simple matrix multiplications.

Note that the last identity in (26), which is also used to deduce the second equation in (25), follows from the definition of matrix $\mathbf{G}$ in (13), which results in $\mathbf{G}^{T} \mathbf{G}=\mathbf{I} \in \mathbb{R}^{n^{g} \times n^{g}}$. Furthermore, $\left(\mathbf{G G}^{T}\right)_{i j}=\delta_{i j}$ if $\boldsymbol{x}_{i} \in \Gamma_{g}$, and 0 otherwise. Consequently, matrix $\mathbf{J}_{g, i j}=\delta_{i j}$ if $\boldsymbol{x}_{i} \notin \Gamma_{g}$, and $\lambda\left(\theta^{2}+\lambda\right)^{-1}$ otherwise. Matrix $\mathbf{J}_{g}$ satisfies $\mathbf{J}_{g} \mathbf{G}=\lambda\left(\theta^{2}+\lambda\right)^{-1} \mathbf{G}$, and thus, for $\lambda=0$, the linear transformation $\mathbf{J}_{g}$ can be understood as the projection of a vector (or system of equations) onto $\operatorname{range}(\mathbf{G})^{\perp}$, that is, onto the components (or degrees of freedom) which have no load contribution from $\mathbf{g}$.

Remark 6. The value of the functional $J_{g}$ at the optimal solution $\mathbf{u}_{n+1}^{B}{ }^{*}$ and $\mathbf{g}_{n+1}^{*}$ satisfying the normal equations in (25) may be expressed as

$$
J_{g}\left(\mathbf{u}_{n+1}^{B}{ }^{*}, \mathbf{g}_{n+1}^{*}\right)=\left\|\mathbf{J}_{g}\left(\mathbf{A} \mathbf{u}_{n+1}^{B}{ }^{*}-\mathbf{b}-(1+\theta) \mathbf{G g}_{n}\right)\right\|^{2}+\lambda\left\|\mathbf{g}_{n+1}^{*}\right\|^{2}
$$

The expression above can be deduced by inserting the expression of $\mathbf{g}_{n+1}$ in $(25)_{2}$ in the definition of $J_{g}$ in $(22)$. Note that $(25)_{1}$ also follows directly from minimising $J_{g}$ in (27). The split form in (25) will also enable us to proof some results regarding the need to regularise the inverse functional $J_{g}$ in $(22)$ :

Proposition 1. If $\lambda=0$ and $\theta>0$, the system of equations in (23) has a unique solution when $\Gamma_{g} \in \Omega^{A}$. Furthermore, if $\Gamma_{g}=\Omega^{A}$, the optimal solution satisfies $J_{g}\left(\mathbf{u}_{n+1}^{B}{ }^{*}, \mathbf{g}_{n+1}^{*}\right)=0$.

Proof. Le us first note that, since $\mathbf{u}^{A}$ prevents solutions with free rigid motions, matrix $\mathbf{A} \in \mathbb{R}^{n \times n^{B}}$ is full-rank, i.e. $\operatorname{kern}(\mathbf{A})=\emptyset \Rightarrow \operatorname{rank}(\mathbf{A})=n^{B}$. Also, in a direct problem with imposed displacements $\mathbf{u}^{A}$, the matrix of the linear system of equations, let us say $\mathbf{A}^{0} \in \mathbb{R}^{n^{B} \times n^{B}}$, is full-rank and formed by matrix $\mathbf{A}$ but with $n^{A}$ rows associated to the dofs in $\mathbf{u}^{A}$ being removed. Therefore $\operatorname{rank}\left(\mathbf{A}^{0}\right)=\operatorname{rank}(\mathbf{A})=n^{B}$.

Due to the form of matrix $\mathbf{J}_{g}$ in (26), we have that when $\lambda=0$ the product $\mathbf{J}_{g} \mathbf{A}$ equals matrix $\mathbf{A}$ but with $n^{g}$ rows set to 0 . If $\Gamma_{g} \in \Omega^{A}$, we have that $n^{g} \leq n^{A}$, and that $G^{h} \subseteq U^{A}$. Therefore, $\operatorname{rank}\left(\mathbf{J}_{g} \mathbf{A}\right)=\operatorname{rank}\left(\mathbf{A}^{0}\right)=n^{B}$, and thus $\mathbf{J}_{g} \mathbf{A} \in \mathbb{R}^{n \times n^{B}}$ is full-rank, which means that the normal equations in (25) have a unique solution. 
When $\Gamma_{g}=\Omega^{A}$, the dimensions of the nodal interpolations satisfy $n^{g}=$ $n^{A}$. In this case, since $\lambda=0$, the system of equations in $(25)_{1}$ reads,

$$
\mathbf{J}_{g} \mathbf{A} \mathbf{u}_{n+1}^{B}=\mathbf{J}_{g} \mathbf{b}
$$

which corresponds to a set of $n$ equations, but with $n^{g}$ of them being trivial statements $0=0$. The remaing $n-n^{g}=n^{B}$ equations correspond to the solution of a direct FE problem with $n^{A}$ prescribed Dirichlet boundary conditions and with matrix $\mathbf{A}^{0}$ specified above. As such, and since it is assumed that the displacements $\mathbf{u}^{A}$ are well-posed, the $n^{B}$ non-trivial equations accept one solution, which is the unique solution of the normal equations in (25). After inserting equation (28) into the definition of $J_{g}$ in (27), the functional vanishes for $\lambda=0$.

\subsection{Contractile forces}

In this section we solve the inverse problem for a material being deformed by contractile dipoles. Therefore, instead of the expression of $J_{g}$ in (22), we will use the following functional,

$$
J_{c}\left(\mathbf{u}_{n+1}^{A}, \mathbf{c}_{n+1}\right)=\left\|\mathbf{K} \mathbf{u}_{n+\theta}+\frac{1}{h} \mathbf{D}\left(\mathbf{u}_{n+1}-\mathbf{u}_{n}\right)-\mathbf{C} \mathbf{c}_{n+\theta}\right\|^{2}+\lambda\left\|\mathbf{c}_{n+1}\right\|^{2} .
$$

The minimisation of $J_{c}\left(\mathbf{u}_{n+1}^{A}, \mathbf{c}_{n+1}\right)$ gives rise to the normal equations,

$$
\begin{array}{r}
\left(\left[\begin{array}{ll}
\mathbf{A} & -\theta \mathbf{C}
\end{array}\right]^{T}\left[\begin{array}{ll}
\mathbf{A} & -\theta \mathbf{C}
\end{array}\right]+\lambda \mathbf{I}_{2}^{T} \mathbf{I}_{2}\right)\left\{\begin{array}{l}
\mathbf{u}_{n+1}^{B} \\
\mathbf{c}_{n+1}
\end{array}\right\} \\
=\left[\begin{array}{ll}
\mathbf{A} & -\theta \mathbf{C}
\end{array}\right]^{T}\left(\mathbf{b}+(1-\theta) \mathbf{C} \mathbf{c}_{n}\right),
\end{array}
$$

with the same matrix definitions given in (24). The normal equations in (30) are the dipole analogous of equations written for nodal loads in (23), where matrix $\mathbf{G}$ has been replaced by $\mathbf{C}$. We also remark that in the present case, and from the definition of $\mathbf{C}$ in (18), we have that

$$
\left(\mathbf{C}^{T} \mathbf{C}\right)_{\alpha \beta}=2 \delta_{\alpha \beta}+\sum_{i} \boldsymbol{e}_{\alpha}^{i T} \boldsymbol{e}_{\beta}^{i}\left(1-\delta_{\alpha \beta}\right) .
$$

Note that the last sum only occurs for one value of $i$, since two element boundaries coincide in at most one node. Therefore $\mathbf{C}^{T} \mathbf{C}$ is a real diagonal dominant matrix, and thus invertible. We can then define a matrix $\mathbf{J}_{c}$ such that,

$$
\mathbf{J}_{c}=\mathbf{I}-\theta^{2} \mathbf{C}\left(\theta^{2} \mathbf{C}^{T} \mathbf{C}+\lambda \mathbf{I}\right)^{-1} \mathbf{C}^{T} .
$$


Remark 7. The solution of the normal equations in (30) is equivalent to solving

$$
\begin{array}{r}
\mathbf{A}^{T} \mathbf{J}_{c} \mathbf{A} \mathbf{u}_{n+1}^{B}=\mathbf{A}^{T} \mathbf{J}_{c}\left(\mathbf{b}-(1-\theta) \mathbf{C} \mathbf{c}_{n}\right) \\
\mathbf{c}_{n+1}=\theta\left(\theta^{2} \mathbf{C}^{T} \mathbf{C}+\lambda \mathbf{I}\right)^{-1} \mathbf{C}^{T}\left(\mathbf{A} \mathbf{u}_{n+1}^{B}-\mathbf{b}-(1-\theta) \mathbf{C} \mathbf{c}_{n}\right)
\end{array}
$$

where matrix $\mathbf{J}_{c}$ is defined in (31).

The form in (32) can been deduced by following the approach detailed in Remark 5. For the case of contractile forces though, the same regularisation requirements stated in Proposition 1 do not hold. Instead, we have the following result:

Proposition 2. If $\lambda=0$ and $\theta>0$, the system of equations in (23) has a unique solution if range $(\mathbf{C}) \cap U^{B}=\emptyset$.

Proof. We will prove the proposition by showing that the matrix in the system of equations $\mathbf{J}_{c} \mathbf{A} \in \mathbb{R}^{n \times n^{B}}$ is full-rank, that is $\operatorname{rank}\left(\mathbf{J}_{c} \mathbf{A}\right)=n^{B}$. To that purpose, we first note that matrix $\mathbf{A}^{0}$, corresponding to matrix $\mathbf{A}$ but removing $n^{A}$ rows associated to dof in $\mathbf{u}^{A}$, has rank $n^{B}$.

We also note that $\mathbf{J}_{c} \mathbf{C}=\lambda \mathbf{C}\left(\theta^{2} \mathbf{C}^{T} \mathbf{C}+\lambda \mathbf{I}\right)^{-1}$, so that when $\lambda=0, \mathbf{J}_{c}$ is the projection onto the orthogonal space spanned by $\mathbf{C}$, that is, $\mathbf{J}_{c}$ projects a vector space onto $\operatorname{range}(\mathbf{C})^{\perp}$. As a projection, and $\operatorname{since} \operatorname{rank}(\mathbf{A})=n^{B}$, we have that

$$
\operatorname{rank}\left(\mathbf{J}_{c} \mathbf{A}\right) \leq n^{B} .
$$

The product $\mathbf{J}_{c} \mathbf{A}$ modifies those rows in range $(\mathbf{C})$. But from the hypothesis of the proposition, range $(\mathbf{C}) \cap U^{B}=\emptyset$, and the fact that $U^{h}=\operatorname{range}(\mathbf{A})=$ $U^{A} \oplus U^{B}$, we have that range $(\mathbf{C}) \subseteq U^{A}$. Consequently, $\mathbf{J}_{c} \mathbf{A}$ does not modify the rows corresponding to the dof in $\Omega^{B}$, and thus $\operatorname{rank}\left(\mathbf{J}_{c} \mathbf{A}\right) \geq \operatorname{rank}\left(\mathbf{A}^{0}\right)=$ $n^{B}$. From this relation and (33) we deduce that $\operatorname{rank}\left(\mathbf{J}_{c} \mathbf{A}\right)=n^{B}$, which indicates that when $\lambda=0$ and $\operatorname{range}(\mathbf{C}) \cap U^{B}=\emptyset, \mathbf{J}_{c} \mathbf{A}$ is not rank deficient.

We remark that the condition range $(\mathbf{C}) \cap U^{B}=\emptyset$ can be ensured in practice by imposing that the dipoles are all applied within the domain $\Omega^{A}$, and that all the $d$ directions of the nodal displacements in $\Omega^{A}$ are known. We also remark that the condition is a sufficient condition for obtaining unique solutions without regularisation, but not necessary. 


\subsection{Determination of regularisation parameter}

The propositions proven above allow us to determine the conditions under which regularisation is not required, at least from the uniqueness standpoint. In the cases where regularisation is required, though they do not give us any estimates of the values of the regularisation parameter $\lambda$. We will here use the L-criterion to determine the optimal of this parameter from the evolution of the error in the equilibrium equations as a function of $\lambda$.

As noted in [22], the use of the L-criterion has some disadvantages. Namely, the difficulty of obtaining optimal values of the regularisation parameter for a smooth solution [15], and the dependence of the criterion on the size of the problem [36]. Alternatively, other methods are equally applicable such as the use of a discrepancy principle [24] or generalised cross-validation principle [37]. However, the sample cases we analyse here have a fixed size and solution's smoothness does not vary in time. For these reasons, and considering the specific error trends we obtain in our analyses, we shall estimate $\lambda$ through the L-criterion.

\section{Numerical Results}

\subsection{Toy problem}

We first analyse a single-element problem in domain $\Omega=[0,1] \times[0,1]$ to illustrate the results in Proposition 1 and 2. We assume that displacements at the two nodes $\boldsymbol{x}_{1}=\{0,0\}^{T}$ and $\boldsymbol{x}_{2}=\{1,0\}^{T}$ have been measured and equal to $\mathbf{u}^{A}=\left\{\mathbf{u}_{1}, \mathbf{u}_{2}\right\}^{T}$ at time $t=h=1.0$ from an undeformed configuration at $t=0$ (see Figure 2a). We then aim to compute the element deformations generated by a known set of contractions (through a direct-method) and apply the inverse method to predict the contractions that have induced these deformations. We do so for three different combinations of loaded nodes: i) nodes $\boldsymbol{x}_{1}$ and $\boldsymbol{x}_{2}$, ii) nodes $\boldsymbol{x}_{1}, \boldsymbol{x}_{2}$ and $\boldsymbol{x}_{3}$, and iii) nodes $\boldsymbol{x}_{1}, \boldsymbol{x}_{2}, \boldsymbol{x}_{3}$ and $\boldsymbol{x}_{4}$. When analysing contractile dipoles, as illustrated in Figure 2a, the three cases correspond to: i) one contraction $c_{1}$ only; ii) two contractions $c_{1}$ and $c_{2}$; and, iii) four contractions $c_{1}, c_{2}, c_{3}$ and $c_{4}$.

In Figure $2 \mathrm{~b}$ we plot the relative error of the retrieved contractions $\mathbf{c}^{*}$ (obtained with the inverse method) with respect to the exact contractions $\mathbf{c}^{\text {exact }}$ (computed through a direct problem), i.e. $r_{c}=\left\|\mathbf{c}_{n+1}^{\text {exact }}-\mathbf{c}_{n+1}^{*}\right\| /\left\|\mathbf{c}_{n+1}^{\text {exact }}\right\|$. It can be observed that in the case of the single contraction, which corresponds 
to the case in Proposition 2, no regularisation is needed and the method provides the exact solution with $\lambda=0$. We have also considered the use of measured displacements $\mathbf{u}^{A}$ with $10 \%$ noise. In this case, the relative error $r_{c}$ is affected by a similar percentage, and an optimal value of regularisation $\lambda \approx 1 E-2$ is found. When considering two and three contractions, the profile of the relative errors show that values with $1 e-10<\lambda<1 e-3$ can be considered without any large variation in the accuracy of the solution.

It should be noted that the presence of noise has minor effects on the solution when $r_{c}$ is larger. However, in those cases when $\operatorname{range}(\mathbf{C}) \cap U^{B} \neq \emptyset$ (e.g. two and three contractions in the toy problem), the minimum relative error may remain large $\left(r_{c} \approx 0.35\right)$ for all ranges of $\lambda$. This fact indicates the importance of taking into account the condition in Proposition 2. Furthermore, it gives rise to variants of the least-squares problem where only the contractions $\mathbf{c}^{B} \in \Omega^{B}$ are regularised. That is, instead of minimising $J_{c}$ in (29), the following functional could be considered,

$$
J_{c}\left(\mathbf{u}_{n+1}^{A}, \mathbf{c}_{n+1}\right)=\left\|\mathbf{K} \mathbf{u}_{n+\theta}+\frac{1}{h} \mathbf{D}\left(\mathbf{u}_{n+1}-\mathbf{u}_{n}\right)-\mathbf{C} \mathbf{c}_{n+\theta}\right\|^{2}+\lambda\left\|\mathbf{c}_{n+1}^{B}\right\|^{2},
$$

with $\mathbf{c}_{n+1}^{B} \in$ range $(\mathbf{C}) \cap U^{B}$. For the toy problem considered here, we have tested that the resulting contractions approach the exact value $\mathbf{c}^{\text {exact }}$ as $\lambda$ increases when no noise is applied. Although in general no exact value may be obtained, partial regularisation would allow capturing the exact surface stress on the measured boundary $\Omega^{A}$.

The visco-elastic material we utilised for this test had Lamé parameters $\lambda=1428$ and $\mu_{L}=357$ (corresponding to Young modulus $E=1000$ and Poisson ratio $\nu=0.4$ ), and $\eta=0.89$. We have numerically tested that the perturbation of those values do not change the trend of the relative error. The relative error of the displacements showed a similar trend.

Tables 1 and 2 list the condition number $\kappa$ and relative errors $r_{g}=\| \mathbf{g}_{n+1}^{*}-$ $\mathbf{g}_{n+1}^{\text {exact }}\|/\| \mathbf{g}_{n+1}^{\text {exact }} \|$ and $r_{c}$ for different cases and values of $\lambda$. It can be verified that case (i) satisfies the sufficient condition in Proposition 1 and 2, and thus the normal equations are well-posed for $\lambda=0$. In this case, the exact solution is recovered, and when using a purely viscous material $(E=0)$, the exact solution is also recovered for $\lambda=1$ (see last rows in Tables 1-2). This is due to the fact that the stress-free isotropic deformation is compatible with the exact solution $\mathbf{g}_{1}=\mathbf{g}_{2}=\mathbf{0}$ (loaded nodes), or $c_{1}=0$ (contractile case), which is also obtained for $\lambda>0$. Cases (ii) and (iii) do not satisfy the necessary 


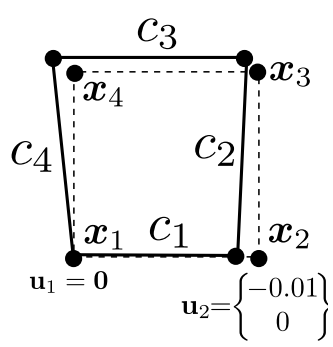

(a)

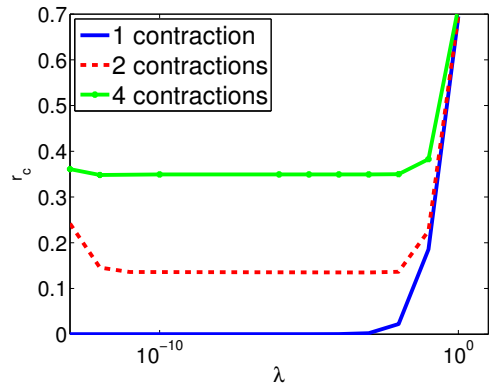

(b)

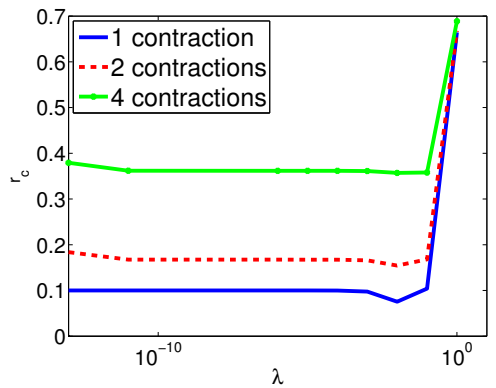

(c)

Figure 2: (a) Toy problem with prescribed displacements and contractions indicated. Displacements are scaled with a factor 5 and correspond to the case with 1 contraction $\left(c_{2}=c_{3}=c_{4}=0\right)$ and $\lambda=0$. (b) Relative error of the computed contractions $r_{c}=\left\|c^{*}-c^{\text {exact }}\right\| /\left\|c^{\text {exact }}\right\|$ vs regularisation parameter $\lambda$ when using no noise in the applied displacements $\mathbf{u}^{A}$. (c) Same plot when using $10 \%$ noise in the applied displacements.

conditions, and for this example the system of equations is ill-posed when $\lambda=0$, and thus require regularisation.

It can be also observed in Tables 1-2 that the condition number of the system matrix in (23) and (30), denoted by $\kappa(\mathbf{N})$ in the tables, is always smaller than $\kappa\left(\mathbf{A}^{T} \mathbf{J}_{g} \mathbf{A}\right)$ or $\kappa\left(\mathbf{A}^{T} \mathbf{J}_{c} \mathbf{A}\right)$, and consequently the alternative system in Remarks 5 and 7 are more stable than the coupled normal equations. In addition, $\kappa\left(\mathbf{A}^{T} \mathbf{J}_{g} \mathbf{A}\right)$ and $\kappa\left(\mathbf{A}^{T} \mathbf{J}_{c} \mathbf{A}\right)$ are not modified when doubling the material stiffness $(E=2000)$, while the matrix of the coupled system is.

The increase of $\lambda$ has beneficial effects on the condition number, overall in cases (ii) and (iii), which are ill-posed for $\lambda=0$. In case (i), increasing $\lambda$ has no effects on the conditioning, but it has detrimental effects in the accuracy of the solution. This example highlights the need to detect problems where no regularisation is needed from the stability standpoint.

In order to verify that the conditions in Proposition 1 and 2 are not necessary, we have used the same measured displacements $\mathbf{u}^{A}$ in nodes $\boldsymbol{x}_{1}$ and $\boldsymbol{x}_{2}$, but retrieved the loads in nodes $\boldsymbol{x}_{2}$ and $\boldsymbol{x}_{3}$ using $\lambda=0$. In this case, the inverse method yields a unique solution, with condition numbers equal to $\kappa(\mathbf{N})=2.56 E-9$ and $\kappa\left(\mathbf{A}_{g}^{\mathbf{J}} \mathbf{A}\right)=4.03 E-4$. Equally, when using contraction $c_{2}$ only and $\lambda=0$, condition numbers are $\kappa(\mathbf{N})=2.12 E-8$ and $\kappa\left(\mathbf{A}^{T} \mathbf{J}_{c} \mathbf{A}\right)=1.08 E-3$, and thus the solution is unique. 


\begin{tabular}{|c|c|c|c|c|c|c|c|c|}
\hline$\lambda$ & $E$ & $\eta$ & Case & $n^{A}$ & $n^{g}$ & $\kappa\left(\mathbf{N}_{g}\right)$ & $\kappa\left(\mathbf{A}^{T} \mathbf{J}_{g} \mathbf{A}\right)$ & $r_{g}$ \\
\hline 0 & 1000 & 0.89 & (i) & 4 & 4 & $8.17 \mathrm{E}-8$ & $5.65 \mathrm{E}-3$ & $2.42 \mathrm{E}-13$ \\
$1 \mathrm{E}-5$ & 1000 & 0.89 & $(\mathrm{i})$ & 4 & 4 & $8.17 \mathrm{E}-8$ & $5.65 \mathrm{E}-3$ & $4.82 \mathrm{E}-5$ \\
1 & 1000 & 0.89 & $(\mathrm{i})$ & 4 & 4 & $2.63 \mathrm{E}-6$ & $2.51 \mathrm{E}-2$ & $8.21 \mathrm{E}-1$ \\
\hline 0 & 1000 & 0.89 & (ii) & 4 & 4 & $1.44 \mathrm{E}-23$ & $6.55 \mathrm{E}-19$ & $6.53 \mathrm{E}+1$ \\
$1 \mathrm{E}-5$ & 1000 & 0.89 & (ii) & 4 & 4 & $2.03 \mathrm{E}-11$ & $2.46 \mathrm{E}-6$ & $2.25 \mathrm{E}-1$ \\
1 & 1000 & 0.89 & (ii) & 4 & 4 & $2.50 \mathrm{E}-6$ & $2.57 \mathrm{E}-2$ & $8.24 \mathrm{E}-1$ \\
\hline 0 & 1000 & 0.89 & (iii) & 4 & 4 & $2.72 \mathrm{E}-23$ & 0.0 & $1.71 \mathrm{E}+2$ \\
$1 \mathrm{E}-5$ & 1000 & 0.89 & (iii) & 4 & 4 & $1.51 \mathrm{E}-11$ & $2.87 \mathrm{E}-2$ & $3.49 \mathrm{E}-1$ \\
1 & 1000 & 0.89 & (iii) & 4 & 4 & $2.28 \mathrm{E}-6$ & $2.87 \mathrm{E}-2$ & $8.27 \mathrm{E}-1$ \\
\hline 0 & 2000 & 0.0 & (i) & 4 & 4 & $2.05 \mathrm{E}-8$ & $5.65 \mathrm{E}-3$ & $2.40 \mathrm{E}-13$ \\
0 & 1000 & 0.0 & (i) & 4 & 4 & $8.17 \mathrm{E}-8$ & $5.65 \mathrm{E}-3$ & $2.05 \mathrm{E}-13$ \\
0 & 0 & 0.89 & (i) & 4 & 4 & $1.16 \mathrm{E}-5$ & $5.43 \mathrm{E}-3$ & $1.20 \mathrm{E}-17$ \\
1 & 1000 & 0.0 & (i) & 4 & 4 & $8.17 \mathrm{E}-8$ & $5.65 \mathrm{E}-3$ & $8.21 \mathrm{E}-1$ \\
1 & 0 & 0.89 & (i) & 4 & 4 & $1.30 \mathrm{E}-5$ & $2.11 \mathrm{E}-2$ & $1.58 \mathrm{E}-18$ \\
\hline
\end{tabular}

Table 1: Condition number $\kappa$ of the system matrix $\mathbf{N}_{g}$ (matrix in parenthesis in equation (23)) and the matrix of the split system in (25) for the three cases analysed (i)-(iii) and different types of materials when using nodal loads (tractions).Last column corresponds to relative error $r_{g}=\| \mathbf{g}_{n+1}^{*}-$ $\mathbf{g}_{n+1}^{\text {exact }}\|/\| \mathbf{g}_{n+1}^{\text {exact }} \|$.

\subsection{Embryo cross-section}

We here apply our methodology to infer the forces driving the deformations of a cluster of cells during morphogenesis of the Drosophila embryo. At the initial instant $t=0$ of our analysis, the in vivo three-dimensional embryo appears to have an almost ellipsoidal shape, whose transverse multiphoton cross-section image is shown in shades of greys in Figure $3[6,7]$. Tissue movements were tracked every $h=45 \mathrm{sec}$ at the nodal locations indicated as orange dots in Figure 3a-c to obtain a complementary displacement field at the remaining interior nodes that form the annulus (not shown in Figure $3 \mathrm{a}-\mathrm{c})$ and the contractions at each cell. Figures 3d-f show the distribution of contractility levels at each cell boundary. To minimise noise and error in tissue tracking, displacement data were smoothed using a Savitzky-Golay filter [27] having a window size of 11 and a smoothing order 3.

We have tested different values of $\lambda$ and using a set of smooth displace- 


\begin{tabular}{|c|c|c|c|c|c|c|c|c|}
\hline$\lambda$ & $E$ & $\eta$ & Case & $n^{A}$ & $n^{c}$ & $\kappa\left(\mathbf{N}_{c}\right)$ & $\kappa\left(\mathbf{A}^{T} \mathbf{J}_{c} \mathbf{A}\right)$ & $r_{c}$ \\
\hline 0 & 1000 & 0.89 & $(\mathrm{i})$ & 4 & 1 & $1.15 \mathrm{E}-6$ & $3.29 \mathrm{E}-2$ & $1.56 \mathrm{E}-15$ \\
$1 \mathrm{e}-5$ & 1000 & 0.89 & $(\mathrm{i})$ & 4 & 1 & $1.15 \mathrm{E}-6$ & $3.29 \mathrm{E}-2$ & $2.280 \mathrm{E}-6$ \\
1 & 1000 & 0.89 & $(\mathrm{i})$ & 4 & 1 & $3.78 \mathrm{E}-6$ & $3.00 \mathrm{E}-2$ & $6.95 \mathrm{E}-1$ \\
\hline 0 & 1000 & 0.89 & (ii) & 4 & 2 & $7.09 \mathrm{E}-22$ & $2.64 \mathrm{E}-17$ & $3.33 \mathrm{E}+1$ \\
$1 \mathrm{e}-5$ & 1000 & 0.89 & (ii) & 4 & 2 & $2.34 \mathrm{E}-11$ & $1.61 \mathrm{E}-6$ & $1.35 \mathrm{E}-1$ \\
1 & 1000 & 0.89 & (ii) & 4 & 2 & $2.52 \mathrm{E}-7$ & $2.65 \mathrm{E}-2$ & $6.98 \mathrm{E}-1$ \\
\hline 0 & 1000 & 0.89 & (iii) & 4 & 4 & $1.72 \mathrm{E}-22$ & $3.56 \mathrm{E}-18$ & $6.08 \mathrm{E}+1$ \\
$1 \mathrm{e}-5$ & 1000 & 0.89 & (iii) & 4 & 4 & $2.07 \mathrm{E}-11$ & $5.22 \mathrm{E}-6$ & $3.49 \mathrm{E}-1$ \\
1 & 1000 & 0.89 & (iii) & 4 & 4 & $2.40 \mathrm{E}-6$ & $3.24 \mathrm{E}-2$ & $7.16 \mathrm{E}-1$ \\
\hline 0 & 2000 & 0.89 & (i) & 4 & 4 & $2.88 \mathrm{E}-7$ & $3.29 \mathrm{E}-2$ & $1.43 \mathrm{E}-15$ \\
0 & 1000 & 0.0 & (i) & 4 & 1 & $1.15 \mathrm{E}-6$ & $3.29 \mathrm{E}-2$ & $3.39 \mathrm{E}-15$ \\
0 & 0.0 & 0.89 & (i) & 4 & 1 & $9.65 \mathrm{E}-6$ & $5.58 \mathrm{E}-2$ & $6.38 \mathrm{E}-18$ \\
1 & 1000 & 0.0 & (i) & 4 & 1 & $3.78 \mathrm{E}-6$ & $3.00 \mathrm{E}-2$ & $6.95 \mathrm{E}-1$ \\
1 & 0.0 & 0.89 & (i) & 4 & 1 & $9.74 \mathrm{E}-6$ & $1.76 \mathrm{E}-2$ & $9.02 \mathrm{E}-19$ \\
\hline
\end{tabular}

Table 2: Condition number $\kappa$ of the system matrix $\mathbf{N}_{c}$ (matrix in parenthesis in equation (30)) and the matrix of the split system in (32) for the three cases analysed (i)-(iii) and different types of materials when using contractility loads (dipoles). Last column corresponds to relative error $r_{c}=\| \mathbf{c}_{n+1}^{\text {exact }}-$ $\mathbf{c}_{n+1}^{*}\|/\| \mathbf{c}_{n+1}^{\text {exact }} \|$.

ments with no noise. Figure 4 shows the profiles of the contracilities at different times for $\lambda=0$ and $\lambda=0.01$. Although the profiles are not affected by $\lambda$, the off-set from the horizontal axis is indeed remarkable when $\lambda=0$. This indicates the ill-conditioning of the matrix in the inverse problem, which dos not satisfy the condition in Proposition 2. We have indeed computed the eig-values of the system, and verified that it contains zero eigen-values, with eigen-vectors associated to the observed off-set. By increasing $\lambda$, the off-set is reduced and contractilities are uniquely defined.

The inverse problem has been also solved using the experimental displacements measured from the cross-sections images. The computed values of the contractilities along the annulus are plotted in Figure 5. The effect of the noise is noticeable on the traction profiles. Furthermore, when $\lambda=0.01$, the general amount of contractility diminishes, in conjunction with a reduction of the off-set from the horizontal level, and a potential transfer of basal 


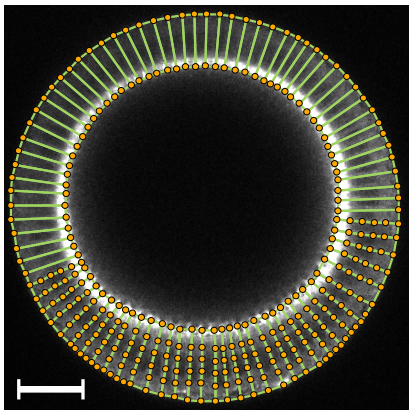

(a) $t=0 \min$

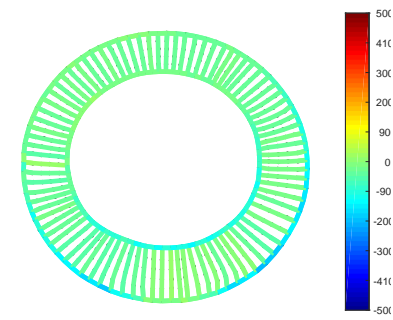

(d) $\lambda=0.01, t=0 \mathrm{~min}$

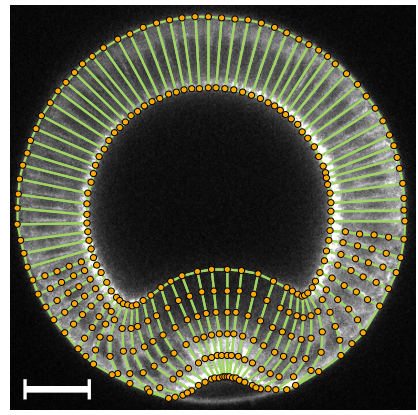

(b) $t=7.5 \mathrm{~min}$

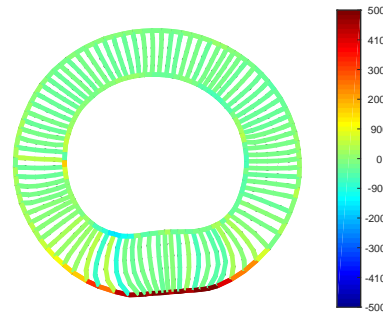

(e) $\lambda=0.01, t=6.75 \mathrm{~min}$

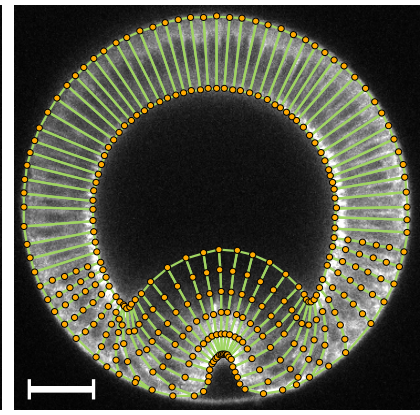

(c) $t=14.25 \mathrm{~min}$

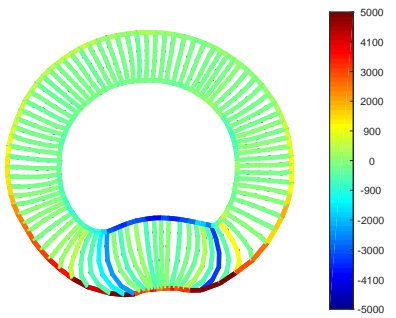

(f) $\lambda=0.01, t=9.75 \mathrm{~min}$

Figure 3: (a-c) Selected multiphoton images of transverse cross-sections of a Drosophila embryo during ventral furrow formation $[6,7]$. The circular cell monolayer is assumed to be a visco-elastic continuum that deforms over time. Measured displacements are indicated through a mesh of fiduciary nodal markers (orange), which follows tissue's deformations. Each cell is subdivided into 5 quadrilateral finite elements. Lateral junctions are indicated in green. Error bars are equal to 20 microns. (d-f) Contractility levels at cell element boundaries (in color code) at indicated time-points and for $\lambda=0.01$.

tractions to the apical (external) side (compare plots in Figures $5 \mathrm{c}$ and $5 \mathrm{f}$ ).

We used a value of the regularisation parameter $\lambda=0.01$, although it can be better determined through the L-criterion by analysing how the error in the visco-elastic equilibrium evolves as a function of $\lambda$. The L-curves for the visco-elastic and purely viscous problems are shown in Figure 6a-b. In all cases, a value $\lambda \approx 0.01-0.1$ is found optimal and close to the point of maximum curvature of the L-curve. 


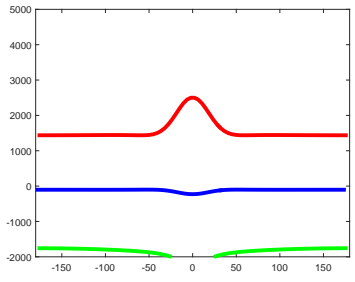

(a) $\lambda=0, t=0 \min$

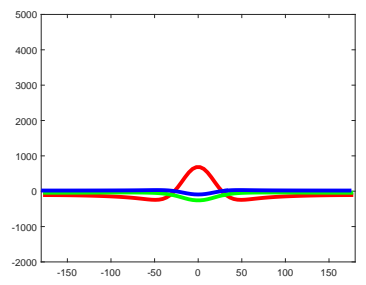

(d) $\lambda=0.01, t=0 \mathrm{~min}$

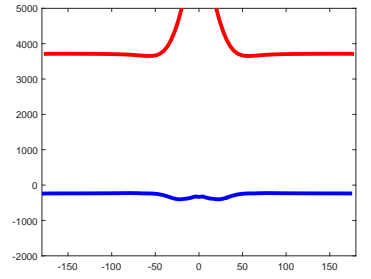

(b) $\lambda=0, t=6.75 \mathrm{~min}$

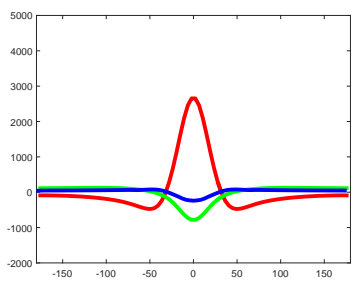

(e) $\lambda=0.01, t=6.75 \mathrm{~min}$

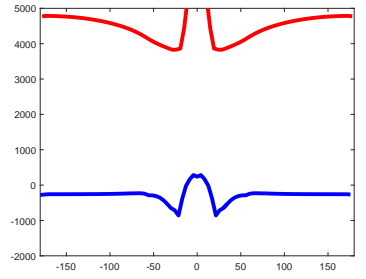

(c) $\lambda=0, t=9.75 \mathrm{~min}$

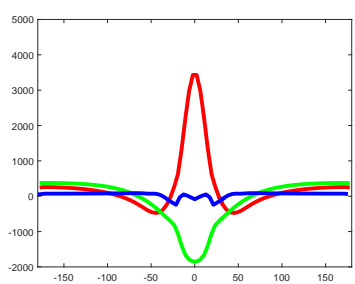

(f) $\lambda=0.01, t=9.75 \mathrm{~min}$

Figure 4: (a-c) Profile of contractilities at three time points for $\lambda=0$ along the circumferential direction (horizontal axis=degrees). Applied displacements have been computed applying a smooth set of contractions and thus contain no noise. Each plot shows contractilities at basal (internal) side (green), at apical (external) side (red), and at lateral sides of cells (blue). (d-f) Contractility profiles for $\lambda=0.01$.

\section{Conclusions}

We have presented a theoretical methodology and its computational implementation to solve an inverse problems for visco-elastic solids. Given a set of measured deformations, this method allows to retrieve either a nodal field of forces at specified locations or a set of contractile dipoles along specified edge lines that determine these visco-elastic deformations.

We have analysed the sufficient conditions that guarantee a unique solution without Tickhonov regularisation for the two loading configurations.

The methodology here presented expands on previous formulations for elastic continua [25] to develop a new approach that extends the results to the case of visco-elastic continua. Differently to a previous visco-elastic formulation of the inverse problem where no equilibrium is imposed in the elastic [13] or visco-elastic case [32], our method minimises a functional that measures the error in the discrete visco-elastic equilibrium in order to satisfy 


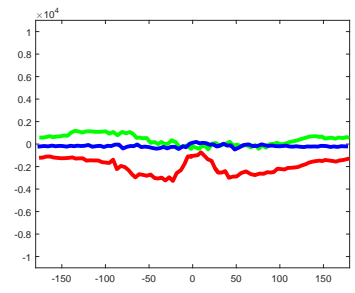

(a) $\lambda=1 E-6, t=0 \min$
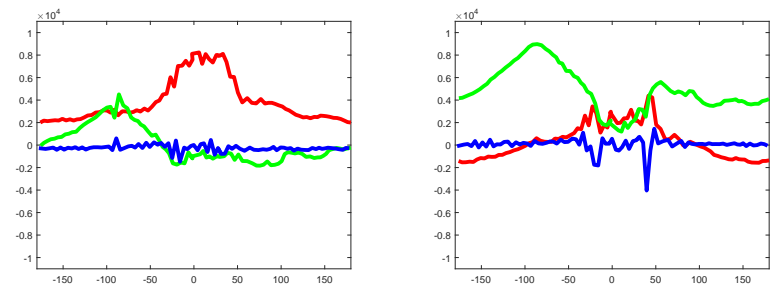

(b) $\lambda=1 E-6, t=6.75$ $\min$

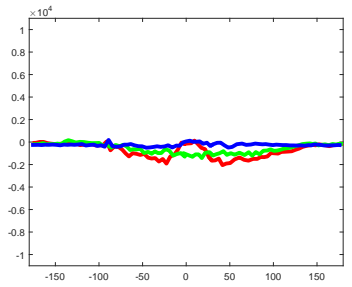

(d) $\lambda=0.01, t=0 \mathrm{~min}$

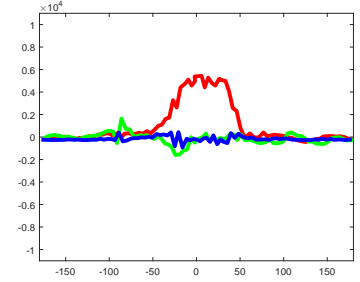

(e) $\lambda=0.01, t=6.75 \mathrm{~min}$ (c) $\lambda=1 E-6, t=9.75$ $\min$

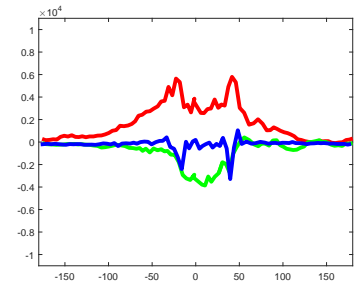

(f) $\lambda=0.01, t=9.75 \mathrm{~min}$

Figure 5: (a-c) Profile of contractilities at three time points for $\lambda=0$ along the circumferential direction (horizontal axis=degrees). Applied displacements have been measured form figures and thus contain experimental noise. Each plot shows contractilities at basal (internal) side (green), at apical (external) side (red), and at lateral sides of cells (blue). (d-f) Contractility values for $\lambda=0.01$.

the quasi-static approximation of motion to which most biological tissues are subjected.

Our results show that the extent of regularisation required to determine a solution of the visco-elastic inverse problem do not depend on the material parameters of the continuum.

Future works will aim at studying the effects of time-variability of the domains $\Omega^{A}$ and $\Omega^{B}$ as well as at formulating approaches for the smoothing of the experimental input and predicted output of the method in order to reduce oscillations in the predictions of displacements $\mathbf{u}_{n+1}^{B}$ and forces $\mathbf{g}_{n+1}$ or $\mathbf{c}_{n+1}$. 


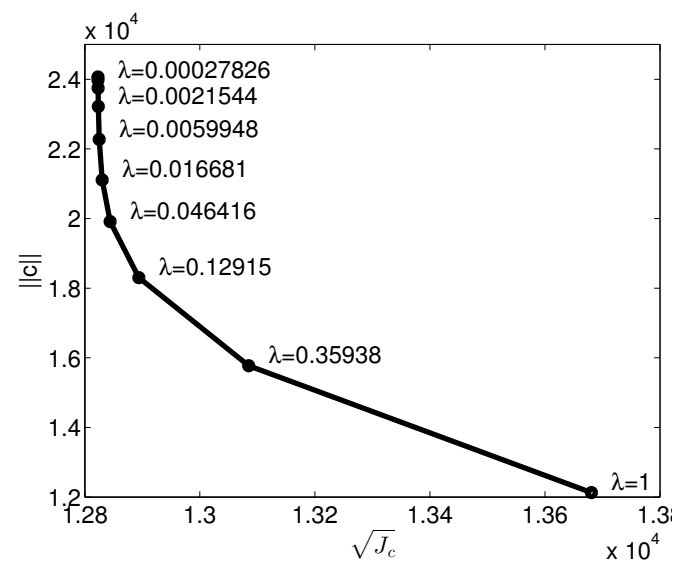

(a)

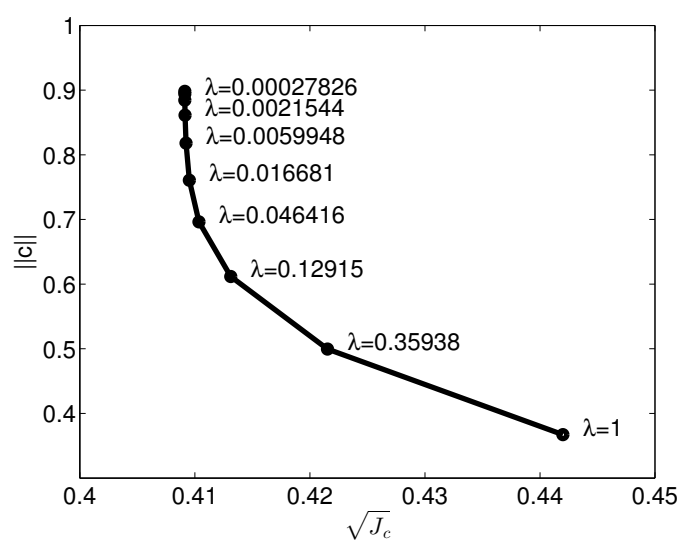

(b)

Figure 6: L-curve for the (a) visco-elastic case, and the (b) purely viscous $\left(\lambda_{L}=\mu_{L}=0, \eta>0\right)$. The purely elastic case $(\eta=0)$ gives a curve that overlaps the curve in (a) for the same values of $\lambda$.

\section{Acknowledgements}

JJM is financially supported by the Spanish Ministry of Industry, Economy and Competitiveness (MINECO) under grants DPI2013-43272-R and DPI2016-74929-R. VC and DA acknowledge support from MINECO through the Centro de Excelencia Severo Ochoa Award to the Institute of Bioengineering of Catalonia (IBEC). VC is financially supported by the MINECO grants BFU2016-75101-P and RYC-2014-15559.

\section{References}

\section{References}

[1] C.J.S. Alves and T. Ha-Duong. Inverse scattering for elastic plane cracks. Inverse Problems, 15:91-97, 1999.

[2] D. Ambrosi. Cellular traction as an inverse problem. SIAM J. Appl. Math., 66(6):2049-2060, 2006. 
[3] S. Andreieux, A. Ben-Abda, and H.D. Bui. Reciprocity principle and crack identification. Inverse Problems, 15:59-65, 1999.

[4] L. Beilina, N. T. Thành, M. V. Klibanov, and M.A. Fiddy. Reconstruction from blind experimental data for an inverse problem for a hyperbolic equation. Inverse Problems, 30:025002, 2014.

[5] M. Bonnet and A. Constantinescu. Inverse problems in elasticity. Inverse Problems, 21:R1-R50, 2005.

[6] G.W. Brodland, V. Conte, P. G. Cranston, J.H. Veldhuis, S. Narasimhan, M.S. Hutson, A. Jacinto, F. Ulrich, B. Baum, and M. Miodownik. Video force microscopy reveals the mechanics of ventral furrow invagination in drosophila. Proc. Natl. Acad. Sci. USA, 107(51):22111-22116, 2010.

[7] G.W. Brodland, J.H. Veldhuis, S. Kim, M. Perrone, D. Mashburn, and M.S. Hutson. Cellfit: A cellular force-inference toolkit using curvilinear cell boundaries. PLoS ONE, 9(6):e99116, 2014.

[8] A. Brugués, E. Anon, V. Conte, J.H. Veldhuis, M. Gupta, J. Collombelli, J.J. Muñoz, G.W. Brodland, B. Ladoux, and X. Trepat. Forces driving epithelial wound healing. Nature Phys., 10:683-690, 2014.

[9] O. Campàs, T. Mammoto, S. Hasso, R.A. Sperling, D. O’Connell, A.G. Bischof, R. Maas, D.A. Weitz, L. Mahadevan, and D.E. Ingber. Quantifying cell-generated mechanical forces within living embryonic tissues. Nature Mat., 11:183-189, 2014.

[10] P.G. Ciarlet. Mathematical Elasticity, volume 1 of Studies in mathematics and its applications. Elsevier Science BV, Amsterdam, 1988.

[11] P. G. Cranston, J.H. Veldhuis, S. Narasimhan, and G. W. Brodland. Cinemechanometry (CMM): A Method to Determine the Forces that Drive Morphogenetic Movements from Time-Lapse Images. Annals Biomed. Engin., 38(9):2937-2947, 2010.

[12] K. Doubrovinski, M. Swan, O. Polyakov, and E.F. Wieschaus. Measurement of cortical elasticity in drosophila melanogaster embryos using ferrofluids. Proc. Natl. Acad. Sci. USA, 114(5):1051-1056, 2017. 
[13] C. Franck, S.A. Maskarinec, D.A. Tirrell, and G. Ravichandran. Threedimensional traction force microscopy: a new tool for quantifying cellmatrix interactions. PLoS ONE, 6(3):e17833, 2011.

[14] E. Grasso, S. Chaillat, J.F. Semblat, and M. Bonnet. Méthode multipôle rapide multi-niveaux en visco-élastodynamique 3D. Giens, France, 2011. 10e Colloque National en Calcul des Structures (CSMA).

[15] M. Hanke. Limitations of the L method in ill-posed problems. BIT Num. Math., 36:287-301, 1996.

[16] B. He, K. Doubrovinski, O. Polyakov, and E. Wieschaus. Apical constriction drives tissue-scale hydrodynamic flow to mediate cell elongation. Nature, 508:392-396, 2014.

[17] C. P. Heisenberg and Y. Bellaïche. Forces in tissue morphogenesis and patterning. Cell, 153(5):948-962, 2013.

[18] J.D. Humphrey, E.R. Dufresne, and M.A. Schwartz. Mechanotransduction and extracellular matrix homeostasis. Nature Reviews Mol. Cell Biol., 15(12):802-812, 2014.

[19] E. Isaacson and H.B. Keller. Analysis of numerical methods. Dover publications, Inc, 1966.

[20] L.E. Malvern. Introduction to the mechanics of continuous medium. Prentice-Hall, Inc., New Jersey, United States of America, 1969.

[21] L. Marin and B.T. Johansson. A relaxation method of an alternating iterative algorithm for the Cauchy problem in linear isotropic elasticity. Comp. Meth. Appl. Mech. Engng., 199:3179-3196, 2015.

[22] L. Marin and D. Lesnic. BEM first-order regularisation method in linear elasticity for boundary identification. Comp. Meth. Appl. Mech. Engng., 192:2059-2071, 2003.

[23] E. Moeendarbary, L. Valon, M. Fritzsche, A.R. Harris, D.A. Moulding, A.J. Thrasher, E. Stride, L. Mahadevan, and G.T. Charras. The cytoplasm of living cells behaves as a poroelastic material. Num. Math., 12:253-261, 2013. 
[24] V.A. Morozov. On the solution of functional equations by the method of regularization. Soviet Math. Dokl., 7:414-417, 1966.

[25] J.J. Muñoz. Non-regularised inverse finite element analysis for 3d traction force microscopy. Int. J. Num. Anal. Mod., 13(5):763-781, 2016.

[26] C. Paleologu, J. Benesty, and S. Ciochină. A robust variable forgetting factor recursive least-squares algorithm for system identification. Signal Proc. Let.., 15:597-600, 2008.

[27] A. Savitzky and M.J.E. Golay. Smoothing and differentiation of data by simplified least squares procedures. Anal. Chem., 36(8):1627-1639, 1964.

[28] U.S. Schwarz, N.Q. Balaban, D. Riveline, A. Bershadsky, B. Geiger, and S.A. Safran. Calculation of forces at focal adhesions from elastic substrate data: the effect of localized force and the need for regularization. Bioph. J., 83:1380-1394, 2002.

[29] X. Serra-Picamal, V. Conte, R. Sunyer, J.J. Muñoz, and X. Trepat. Mapping forces and kinematics during collective cell migration. Meth. Cell Biol., 125:309-330, 2015.

[30] K. Sugimura, P.F. Lenne, and F. Graner. Measuring forces and stresses in situ in living tissues. Development, 143:186-196, 2016.

[31] R. Sunyer, V. Conte, J. Escribano, A. Elosegui-Artola, A. Labernadie, L. Valon, D. Navajas, J.M. García-Aznar, J.J. Muñoz, P. Roca-Cusachs, and X. Trepat. Collective cell durotaxis emerges from long-range intercellular force transmission. Science, 353(6304):1157-1161, 2016.

[32] J. Toyjanova, E. Hannen, E. Bar-Kochba, E.M. Darling, D.L. Henanna, and C. Franck. 3D viscoelastic traction force microscopy. Soft Matter, 10:8095-8106, 2014.

[33] J.H. Veldhuis, A. Ehsandar, J.L Maître, T. Hiiragi, S. Cox, and G. W. Brodland. Inferring cellular forces from image stacks. 372(1720):20160261, 2017.

[34] G. Vitale, L. Preziosi, and D. Ambrosi. Force traction microscopy: An inverse problem with pointwise observations. J. Math. Anal. Appl., 395:788-801, 2012. 
[35] G. Vitale, L. Preziosi, and D. Ambrosi. A numerical method for the inverse problem of cell traction in 3D. Inverse Problems, 28:095013, 2012 .

[36] C.R. Vogel. Non-convergence of the L-curve regularization parameter method. Inverse Problems, 12:535-547, 1996.

[37] G. Wahba. Practical approximate solution to linear operator equations when the data is noisy. SIAM J. Num. Anal., 14:651-667, 1977.

[38] M.A. Wozniak and C.S. Chen. Mechanotransduction in development: a growing role for contractility. Nature Reviews Mol. Cell Biol., 10:34-43, 2009.

[39] W.C. Yeih, T. Koya, and T. Mura. An inverse problem in elasticity with partially overspecied boundary conditions. i. theoretical approach. Trans. ASME J. Appl. Mech., 60:595-600, 1993. 